\title{
Mechanical and electro-mechanical properties of three-dimensional nanoporous graphene-poly(vinylidene fluoride) composites
}

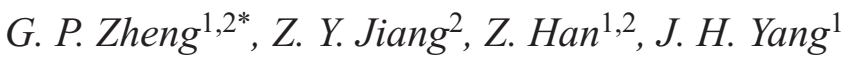 \\ ${ }^{1}$ School of Materials Science and Engineering, University of Shanghai for Science and Technology, Shanghai 200093, \\ China \\ ${ }^{2}$ Department of Mechanical Engineering, Hong Kong Polytechnic University, Hung Hom, Kowloon, Hong Kong, China
}

Received 13 January 2016; accepted in revised form 28 March 2016

\begin{abstract}
Three-dimensional nanoporous graphene monoliths are utilized to prepare graphene-poly(vinylidene fluoride) nanocomposites with enhanced mechanical and electro-mechanical properties. Pre-treatment of the polymer (poly(vinylidene fluoride), PVDF) with graphene oxides (GOs) facilitates the formation of uniform and thin PVDF films with a typical thickness below $100 \mathrm{~nm}$ well coated at the graphene nano-sheets. Besides their excellent compressibility, ductility and mechanical strength, the nanoporous graphene-PVDF nanocomposites are found to possess high sensitivity in strain-dependent electrical conductivity. The improved mechanical and electro-mechanical properties are ascribed to the enhanced crystalline $\beta$ phase in PVDF which possesses piezoelectricity. The mechanical relaxation analyses on the interfaces between graphene and PVDF reveal that the improved mechanical and electro-mechanical properties could result from the interaction between the $-\mathrm{C}=\mathrm{O}$ groups in the nanoporous graphene and the $-\mathrm{CF}_{2}$ groups in PVDF, which also explains the important role of GOs in the preparation of the graphene-polymer nanocomposites with superior combined mechanical and electro-mechanical properties.
\end{abstract}

Keywords: processing technology, nanoporous graphene, ferroelectric polymers, mechanical properties, dynamic mechanical relaxation

\section{Introduction}

In the past several years three-dimensional (3D) nanoporous graphene structures have attracted much attention since they are promising multi-functional materials which implement the superior properties of two-dimensional (2D) graphene sheets for macroscopic applications [1-5]. Graphene aerogel (GA) is the most typical 3D network structure consisting of graphene nano-sheets and possesses ultra-light weight (density as low as $0.16 \mathrm{mg} / \mathrm{cm}^{3}$ ), ultra-high surface area (as large as $600 \mathrm{~m}^{2} / \mathrm{g}$ ), super-elasticity, high compressibility and conductivity. They could be used as mechanical dampers, strain gauge, stress and pressure sensors with high performances [6-10]. However their weak inter-connected graphene structures have prevented GAs from exhibiting stable mechanical and electro-mechanical behaviours. For example, the mechanical strength $(<100 \mathrm{kPa})$ and Young's modulus $(<5 \mathrm{MPa})$ of GA are too low for practical functional application [1-3]. In particular, formation of micro-cracks inside GAs after cyclic loading could deteriorate their mechanical and electro-mechanical performances.

In this work, we develop GA supported poly(vinylidene fluoride) (GAsPVDF) polymers which could be promising multi-functional materials. Poly(vinylidene fluoride) (PVDF) has been widely used as functional or structural material in water-proof layers, 
sensors, transducers and actuators. PVDF is a semicrystalline polymer and is typically brittle. When it is used for piezoelectric or pyroelectric applications, the desirable content of polar crystalline $\beta$ phase in PVDF has to be developed through mechanical deformation or electrical poling, and is difficult to be controlled. Hence, PVDF polymers used as sensors or actuators are typically micro-sized thin films and bulk PVDF is usually not much useful in piezoelectric and pyroelectric applications. Besides their high mechanical strength, excellent compressibility and ductility, a hybrid structure of GA and PVDF films is expected to exhibit superior dielectric, piezoelectric and pyroelectric properties because of the ultra-high surface areas of the adsorbed thin PVDF films, and could be better functional materials as compared to GA and PVDF [11]. Although functionalization of GA with oxides, polymers and metals has been reported in previous studies [12-16], this work is the first attempt in engineering GA with dielectric and ferroelectric materials. The bulk GA functionalized with PVDF films could exhibit superior combined mechanical and electro-mechanical properties and is versatile for various functional applications.

Although the GAs exhibit great hydrophobicity because of their intrinsically large surface area and nonpolar carbon structure, for organic solvents and oils, they have excellent adsorption capacity and ultrafast absorption rate. Based on these features, GAs can be immersed in organic solvent to form composite structures with other functional materials. In this work, we synthesize GAsPVDF multi-functional materials via immersing GA in organic solutions of PVDF. Since the $-\mathrm{CH}_{2}$ and $-\mathrm{CF}_{2}$ groups in $\mathrm{PVDF}$ are active toward some functional molecular groups which exist more or less in GA (usually reduced graphene oxides or RGO), it is expected that the main chains of PVDF are attracted to the graphene sheets in GA. On the other hand, blending of PVDF with graphene oxides (GOs) in organic solution has been found to result in a PVDF-GO nanocomposite with enhanced ferroelectric and pyroelectric properties [17, 18]. In the formation of GAsPVDF, we add GOs into the organic solution of PVDF where GA is immersed. GOs could not only act as surfactants of GA but also facilitate the coating of PVDF-GO nanocomposites with enhanced piezoelectric and ferroelectric properties on the graphene nano-sheets. Comparative studies are carried out to investigate the mechanical and electromechanical properties of GAsPVDF samples prepared with and without GO blending of PVDF solutions. X-ray diffraction (XRD), scanning electron microscopy (SEM), Fourier-transform infra-red (FTIR) and Raman spectroscopy are used to analyze the structural properties of the GAsPVDF samples prepared by different methods. Samples are tested under compression to characterize their mechanical properties. The relation between the compressive strain and electrical resistance of GAsPVDF are measured to characterize the electro-mechanical properties of the samples. The effects of graphene and GO sheets on the formation of PVDF films inside GA are analyzed using anelastic mechanical relaxation, which is effective in analyzing the interaction between the nano-fillers (PVDF) and the porous matrix (GA) [19]. The interaction between the functional molecular groups in GAs or GOs and $-\mathrm{CF}_{2}$ groups in PVDF is characterized to elucidate the atomistic behaviors of the graphene-PVDF hybrid structures inside GAs.

\section{Experimental methods}

GOs were chemically exfoliated from natural graphite flakes via a modified Hummers method [20]. Graphite flakes (5.0 g) (CP, Aladdin Reagent Database Inc., Shanghai, China) and sodium nitrates $(2.5 \mathrm{~g})(\mathrm{CP}, \mathrm{Al}-$ addin Reagent Database Inc., Shanghai, China) were added into concentrated sulfuric acid $(115 \mathrm{~mL})$ at room temperature. After soaking in ice bath for 25 mins with mild agitation, potassium permanganate (15.0 g) (CP, Aladdin Reagent Database Inc., Shanghai, China) was added gradually into the mixture. Then, the mixed suspension was heated to $35^{\circ} \mathrm{C}$ and kept for $45 \mathrm{~min}$. Deionized water $(230 \mathrm{~mL})$ was added and the temperature of the solution was kept at $98^{\circ} \mathrm{C}$ for 45 mins. When the brown mixture turned into yellow, the mixture solution was diluted to $700 \mathrm{~mL}$, followed by adding $30 \mathrm{~mL} \mathrm{H}_{2} \mathrm{O}_{2}(30 \%)$. The mixture was then filtered and washed with $50 \mathrm{~mL}$ of $\mathrm{HCl}$ solution $(9 \%)$. Finally, the solution was centrifuged for several times at $11000 \mathrm{rpm}$ until the $\mathrm{pH}$ of the system was about 7 . The resulting sample was dried in vacuum at $60^{\circ} \mathrm{C}$ for $72 \mathrm{~h}$.

GO solution of $2 \mathrm{mg} \cdot \mathrm{mL}^{-1}$ was prepared by the sonication of $2.2 \mathrm{~g}$ graphite oxide in $1 \mathrm{~L}$ water for about 2 hours. The aggregates were then removed by mild centrifugation (3000 rpm for 10 mins). Graphene hy- 
drogels (GHs) were fabricated by a typical hydrothermal method. $2 \mathrm{mg} \cdot \mathrm{mL}^{-1}$ of $\mathrm{GO}$ solution was put in a Teflon-lined stainless-steel autoclave, sealed, and hydrothermally treated at $180^{\circ} \mathrm{C}$ for $20 \mathrm{~h}$ to obtain $\mathrm{GH}$. Then GH was further hydrothermally treated in ammonia solution at $90^{\circ} \mathrm{C}$ for $1 \mathrm{~h}$. After freeze drying, the cylindrical GA with a typical height of $10 \mathrm{~mm}$ was obtained. The diameter of the $3 \mathrm{D}$ graphene monoliths is $5-8 \mathrm{~mm}$.

GAsPVDF samples were prepared via immersing GAs directly in N,N-dimethylformamide (DMF, SigmaAldrich, Shanghai, China) with different concentrations of PVDF (Sigma-Aldrich, Shanghai, China). The schematic of the preparation process is shown in Figure 1. The concentrations of PVDF in the DMF solvent are measured by the mass ratio of PVDF to DMF. The procedure of preparing GAsPVDF with GO blending in the PVDF solution is described as follows. First, the graphene oxides were dissolved in DMF with the assistance of sonication and agitation. Then the PVDF powder was added to the suspension and stirred for 8 hours. The mass ratio of PVDF to GO was kept as a constant (50:1) in the solutions with different concentrations of PVDF. Finally, the cylindrical GAs were immersed in the solutions under sonication for 3 hours and held for another 24 hours for the complete impregnation of GAs with the solution. The GAsPVDF samples were obtained after the samples were dried at $70^{\circ} \mathrm{C}$ in a vacuum oven till the DMF was removed completely. The samples are denoted as GAsPVDF-GOx where $x=0.5,1,3$ and $10 \%$ represent the concentrations of PVDF in the DMF solvent. Similar steps were carried out to prepare GAsPVDF without GO blending in the PVDF solution. The synthesized samples are denoted as GAsPVDF- $x$ where $x=1,2,3$ and $10 \%$ represent the concentrations of PVDF in the DMF solvent.

Functional groups and chemical bonds of the synthesized samples were determined by X-ray photoelectron spectroscopy (XPS, PHI 5000C ESCA, Perkin Elmer, Massachusetts, USA) at a base pressure of $1 \cdot 10^{-9}$ mbar. XRD patterns of the samples were taken by an X-ray diffractometer (XRD, PW3040/60, Philips, Netherlands) with nickel filtered $\mathrm{Cu} \mathrm{K} \alpha$ radiation. The microstructures of samples were investigated by Scanning Electron Microscopy (SEM, JSM6490, JEOL Inc., Peabody, USA) which was equipped with energy dispersive X-ray (EDX) analysis and was operated at $20 \mathrm{kV}$. FT-IR spectra were recorded by an FT-IR spectrophotometer (Spectrum 100, Perkin Elmer, Massachusetts, USA) with a resolution of $1.0 \mathrm{~cm}^{-1}$. Raman experiments were carried out on a Raman Station (Model 400/400F, Perkin Elmer, Massachusetts, USA) with a resolution of $1.0 \mathrm{~cm}^{-1}$. The compressive curves of the samples were measured by a compression testing machine (Materials Testing Z2.5TH, Zwick Techonology \& Instrument Co. Ltd, Shanghai, China). The samples were pol-

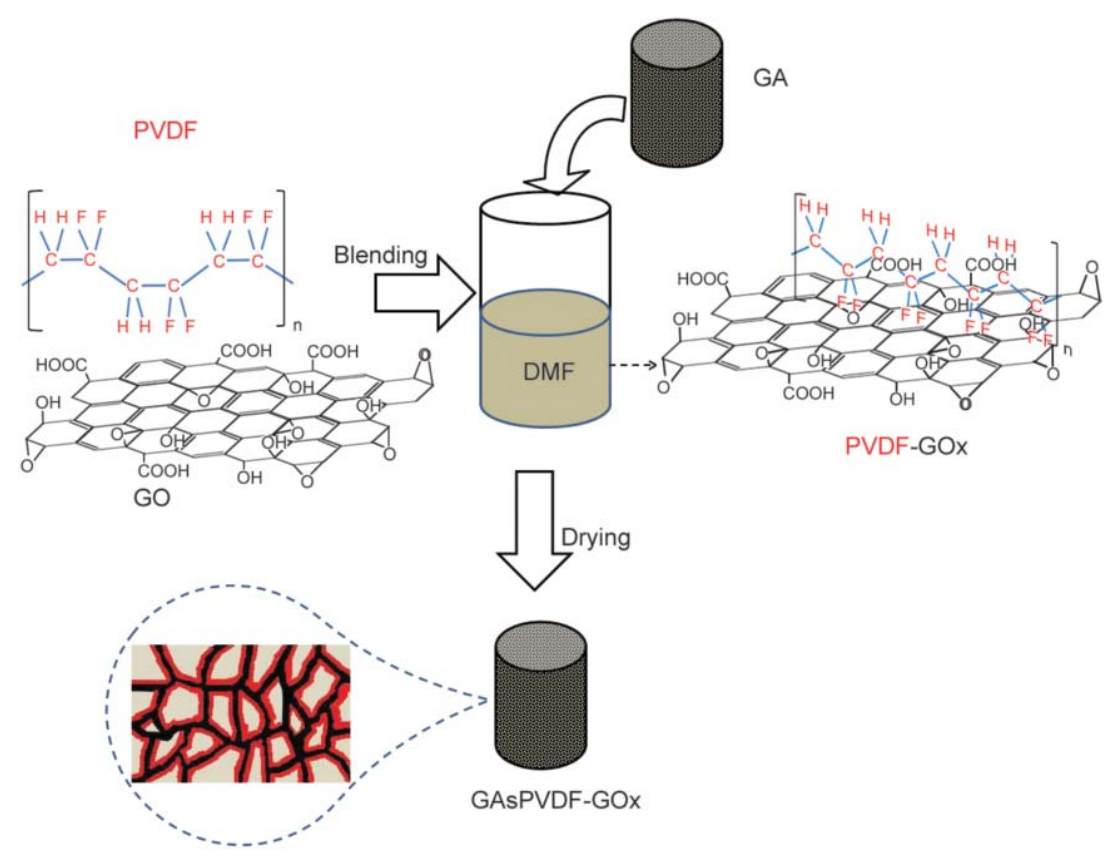

Figure 1. Schematic of preparation of GAsPVDF-GOx from PVDF solution with GO blending. The microstructures of the resulting GAsPVDF-GOx samples are illustrated as PVDF films (in red) coated at the graphene sheets (in black) 
ished into a cylindrical shape with a height of $8 \mathrm{~mm}$ and a diameter of $4 \mathrm{~mm}$. The compressive strain rate was $0.02 \mathrm{~min}^{-1}$. Samples with the same dimensions were used for electrical resistance measurement under compression. Silver-paste layers with a thickness of $0.1 \mathrm{~mm}$ were coated at the top and bottom surfaces of the samples to form electrodes. The ferroelectric test system (TF2000E, aixACCT, Aachen, Germany) under the leakage current measuring mode was used to measure the currents of the sample under the applied voltages of $0-2 \mathrm{~V}$. The electrical resistance was calculated from the Ohm's law.

Mechanical relaxation of graphene sheets and graphene-PVDF interfaces in GAsPVDF samples were characterized using a Dynamic Mechanical Analyzer (DMA, Q800, TA Instruments, Minneapolis, USA). The samples were polished into a cylindrical shape with a height of $10 \mathrm{~mm}$ and a diameter of $5 \mathrm{~mm}$ and were tested in a compression mode of dynamic mechanical analysis. The sinusoidal strain with an amplitude of $0.3 \%$ at various frequencies were applied to the samples. The anelastic relaxation behaviors of the samples between 50 and $-150^{\circ} \mathrm{C}$ were measured. The cooling rate is $0.6^{\circ} \mathrm{C} / \mathrm{min}$.

\section{Results and discussion}

\subsection{Characterizations of GA supported poly(vinylidene fluoride)}

SEM images of the GAsPVDF-GOx samples are shown in Figure 2. In the preparation of GAsPVDFGOx with low concentrations of PVDF ( $x=0.5$ and $1 \%$ ), a relatively small amount of PVDF and GOs are dissolved in the DMF solvent. Since the solution is a very dilute liquid and there is strong interaction between PVDF and GO, PVDF tends to form particulates with GOs rather than coatings on the graphene sheets inside GAs. As shown in the SEM images (Figure 2a, b), small PVDF-GO particles only cover surfaces of the graphene sheets inside GAs. With increasing PVDF and GO contents in the DMF solvent $(x=3$ and 10\%), the PVDF solution becomes more viscous and more PVDF and GO are absorbed into GAs. As shown in Figure 2c, d, film-like PVDF is adsorbed much easier into GAs immersed in the viscous PVDF solution. The red arrows in Figure $2 d$ are used to estimate the thickness of PVDF-absorbed graphene nanosheets, which is about $90 \mathrm{~nm}$. Thus it can be determined that the thickness of PVDF films
Table 1. Molar fraction of $\mathrm{F}$ atoms in GAsPVDF-GOx samples determined from XPS and EDX analyses

\begin{tabular}{|c|c|c|c|c|}
\hline & $\boldsymbol{x}=\mathbf{0 . 5 \%}$ & $\boldsymbol{x}=\mathbf{1 \%}$ & $\boldsymbol{x}=\mathbf{3 \%}$ & $\boldsymbol{x}=\mathbf{1 0} \%$ \\
\hline $\mathrm{XPS}$ & $0.098 \pm 0.001$ & $0.102 \pm 0.001$ & $0.144 \pm 0.001$ & $0.212 \pm 0.001$ \\
\hline $\mathrm{EDX}$ & $0.083 \pm 0.005$ & $0.097 \pm 0.005$ & $0.146 \pm 0.005$ & $0.209 \pm 0.005$ \\
\hline
\end{tabular}

in the GAsPVDF-GO10\% sample could be smaller than $100 \mathrm{~nm}$. EDX analysis is used to determine the molar fraction of $\mathrm{F}$ atoms relative to the total atoms $(\mathrm{C}, \mathrm{O}, \mathrm{F})$ in the GAsPVDF-GOx samples. Table 1 shows the relation between $\mathrm{x}$ and the molar fraction of $\mathrm{F}$ atom which could be proportional to the PVDF content in GA. The PVDF content of GAsPVDFGOx is found to increase with increasing PVDF concentration of the solution.

SEM images of the GAsPVDF-x samples are shown in Figure 3. The PVDF forms a network structure based on the architecture of graphene aerogel. The concentration of PVDF solution $(x=1,2,3,10 \%)$ does not much affect the morphology of PVDF formed inside GAs. The thickness of PVDF is found to be much thicker than that of GAsPVDF-GOx. Comparison on the SEM images of GA (Figure 2e), GAsPVDF-GO10\% (Figure 2d) and GAsPVDF-10\% (Figure 3d), demonstrates that GO blending in the PVDF solution facilitates the formation of uniform and thin PVDF coatings on the graphene sheets inside GA.

XPS spectrum shown in Figure 4 indicates that the GAsPVDF-GOx samples have a predominant $F$ 1s peak at around $663 \mathrm{eV}$. The content of PVDF in the GAsPVDF-GOx samples could be characterized by the molar fraction of $\mathrm{F}$ atoms in the samples consisting of $\mathrm{C}, \mathrm{O}$ and $\mathrm{F}$ atoms. As listed in Table 1, it is found that the molar fraction of $F$ atoms in the GAsPVDF-GOx sample determined by XPS increases with increasing concentration of PVDF in the solution and is close to that determined by EDX, especially in the GAsPVDF-GOx samples with $x>$ $1 \%$. The quantitative comparison on the molar fractions of $\mathrm{F}$ atoms determined by EDX and XPS suggests that the PVDF thin films could be coated uniformly on the graphene sheets in the GAsPVDFGOx samples.

XRD patterns of the GAsPVDF samples prepared from PVDF solution with and without GO blending are shown in Figure 5. The intensities of the XRD peaks at $20.4^{\circ}$ corresponding to the (110) planes of 


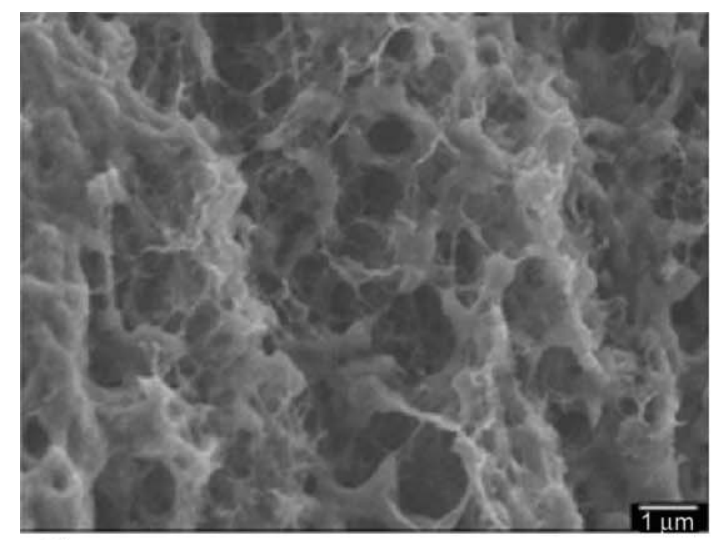

a)

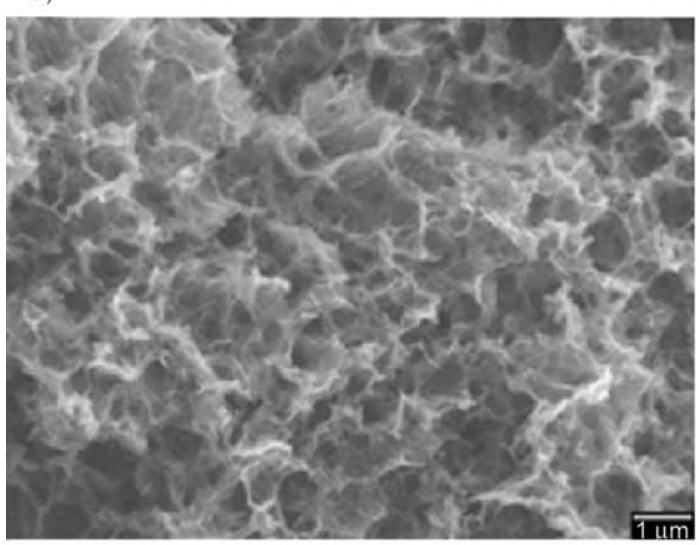

c)

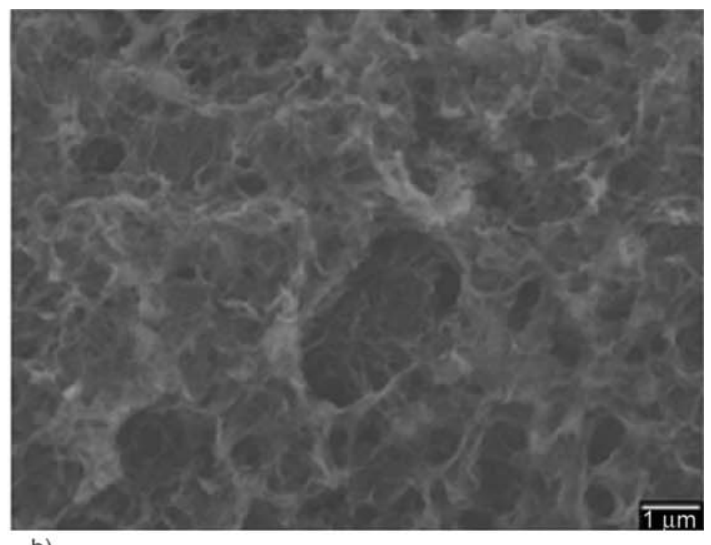

b)

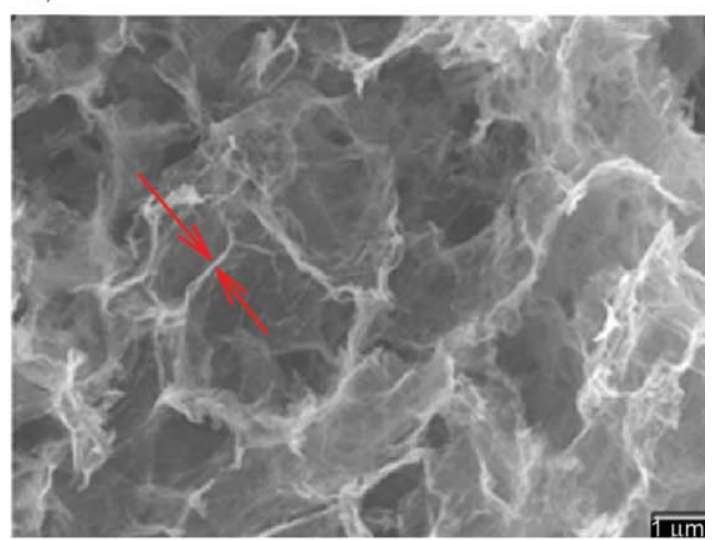

d)

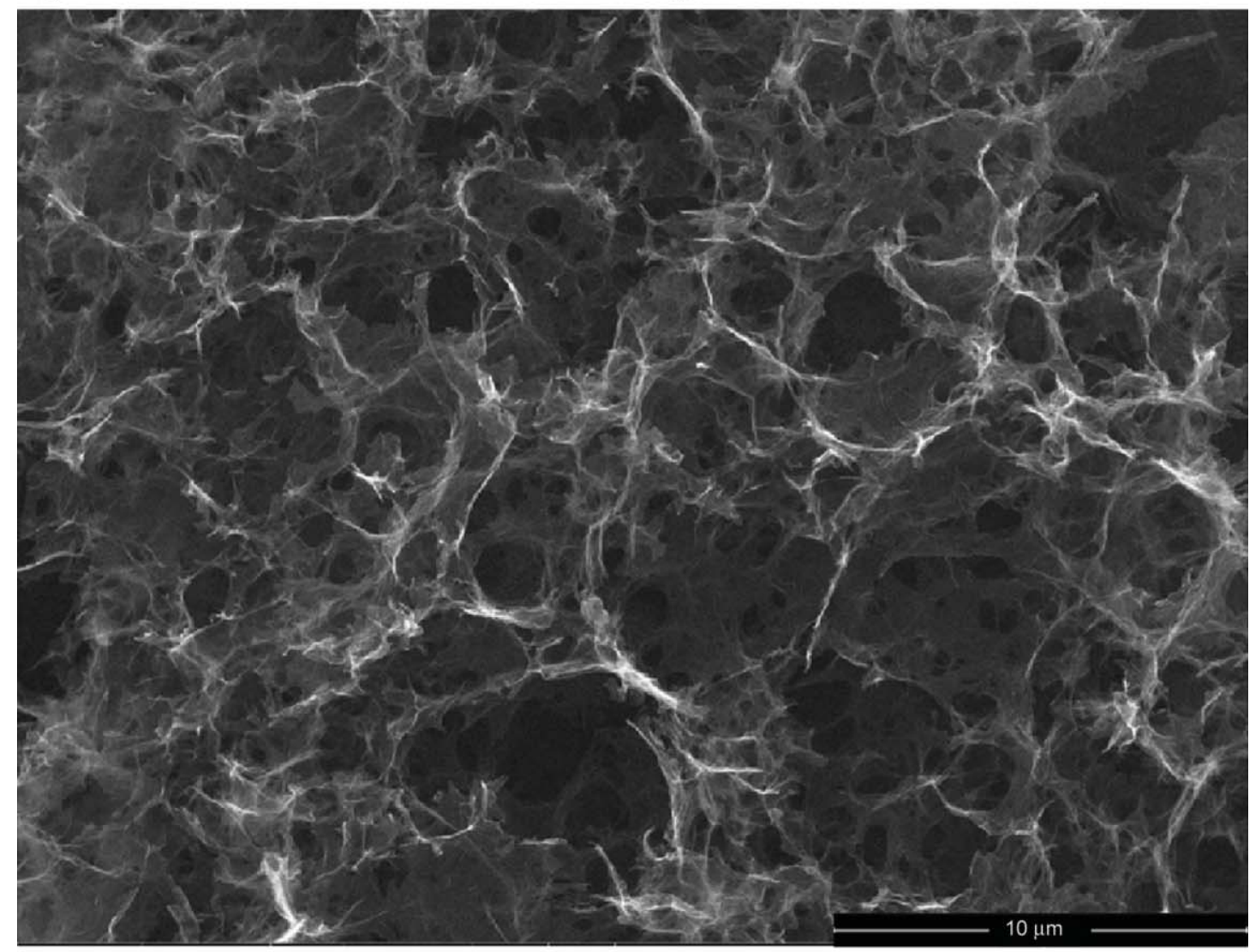

e)

Figure 2. SEM images of GAsPVDF-GOx samples prepared from PVDF solution with GO blending. (a)-(d): $x=0.5,1,3$, $10 \%$, respectively. (e) GA. The arrows in (d) are used to estimate the thickness of PVDF-absorbed graphene nanosheets. 


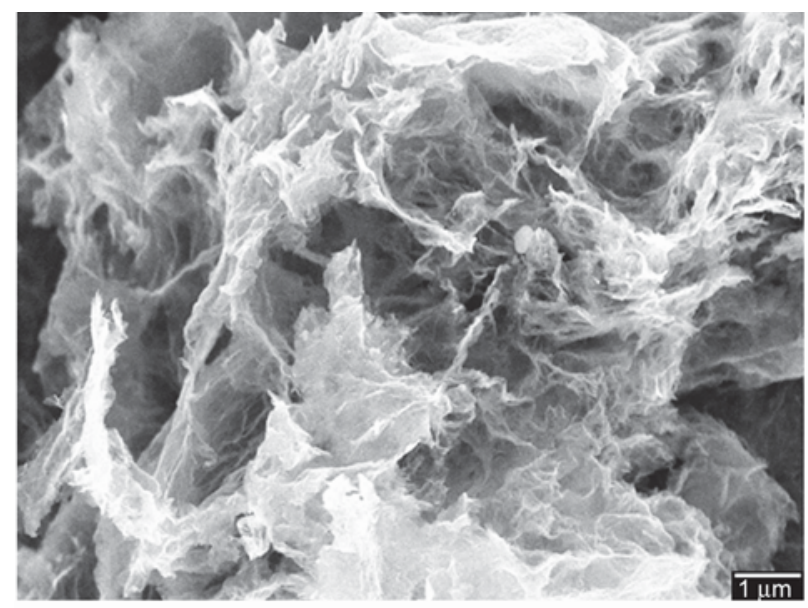

a)

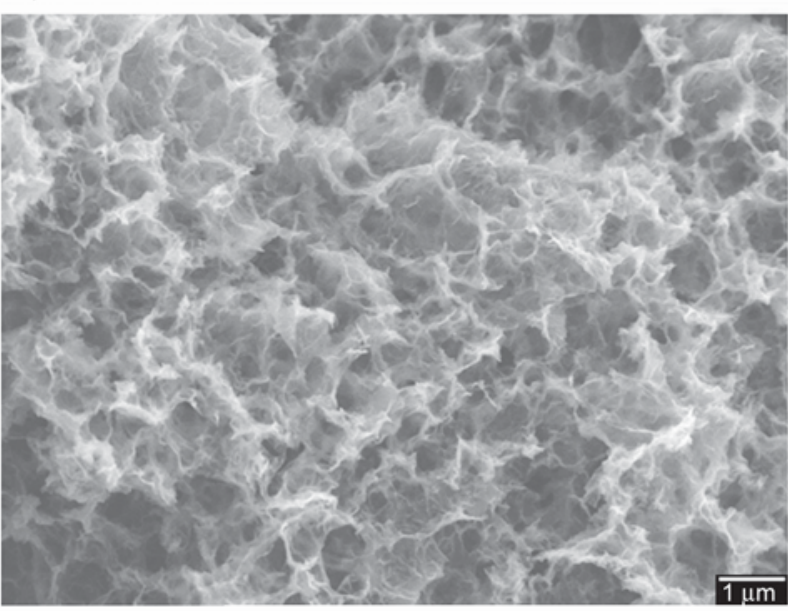

c)

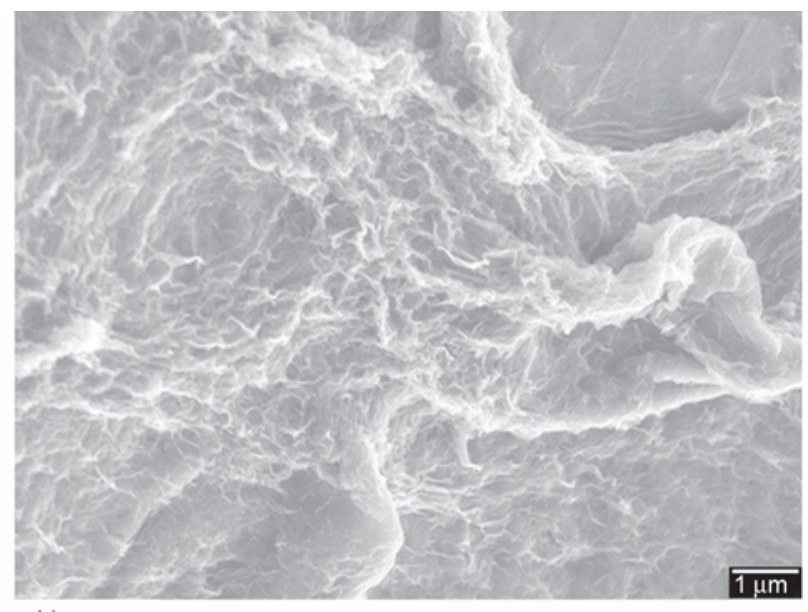

b)

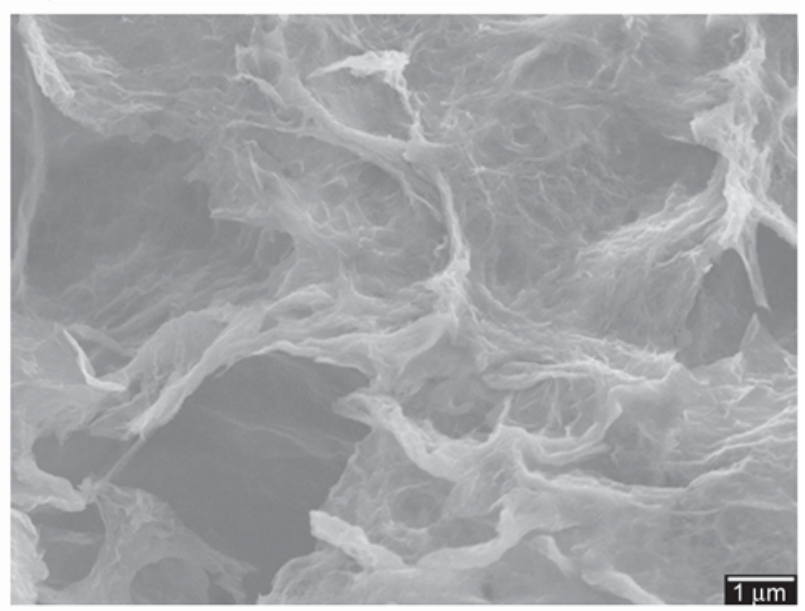

d)

Figure 3. SEM images of GAsPVDF-x samples prepared from PVDF solution without GO blending. (a) $x=1 \%$; (b) $x=2 \%$; (c) $x=3 \%$; (d) $x=10 \%$.

the crystalline $\beta$ phase of PVDF increase with increasing PVDF content $x$. Furthermore, the GAsPVDFGOx samples are found to have stronger (110) peak of PVDF $\beta$ phase (relative to the (001) peak of GA at $24.6^{\circ}$ ) than that of the GAsPVDF-x samples.

FTIR spectra of the GAsPVDF-GOx samples prepared from PVDF solution with GO blending are

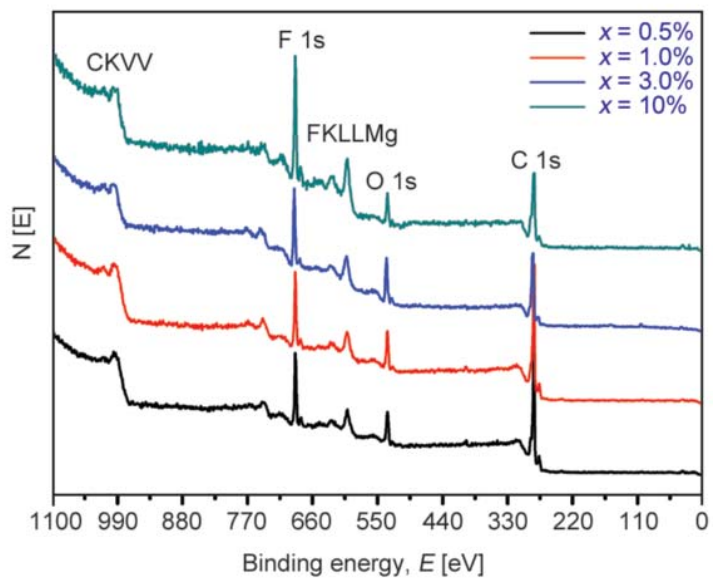

Figure 4. XPS survey scan of GAsPVDF-GOx samples shown in Figure 6a. The IR transmittance peak at $839 \mathrm{~cm}^{-1}$ characterizes the presence of the $\beta$-polymorph phase in PVDF. In the $\beta$ conformation of PVDF, fluorine and hydrogen atoms are on opposite sides of the main molecule chains and hence the net non-zero dipole moments are formed. With increasing content of PVDF, the transmission intensity at $839 \mathrm{~cm}^{-1}$ becomes stronger, demonstrating that the crystalline $\beta$ phase of PVDF could increase with increasing concentration of PVDF.

Raman spectra of the GAsPVDF-GOx samples prepared from PVDF solution with GO blending are shown in Figure 6b. The D-band $\left(1327 \mathrm{~cm}^{-1}\right)$ represents the defects in the graphene or amorphous carbon; the G-band $\left(1593 \mathrm{~cm}^{-1}\right)$ is caused by the inplane vibration of graphite with an $E_{2 \mathrm{~g}}$-symmetry intra-layer mode. The intensity ratio of G-band to Dbands $\left(I_{\mathrm{G}} / I_{\mathrm{D}}\right)$ is much higher in GAsPVDF-GO10\% than any other samples, suggesting that the defective carbon structures in GAsPVDF-GOx are repaired 


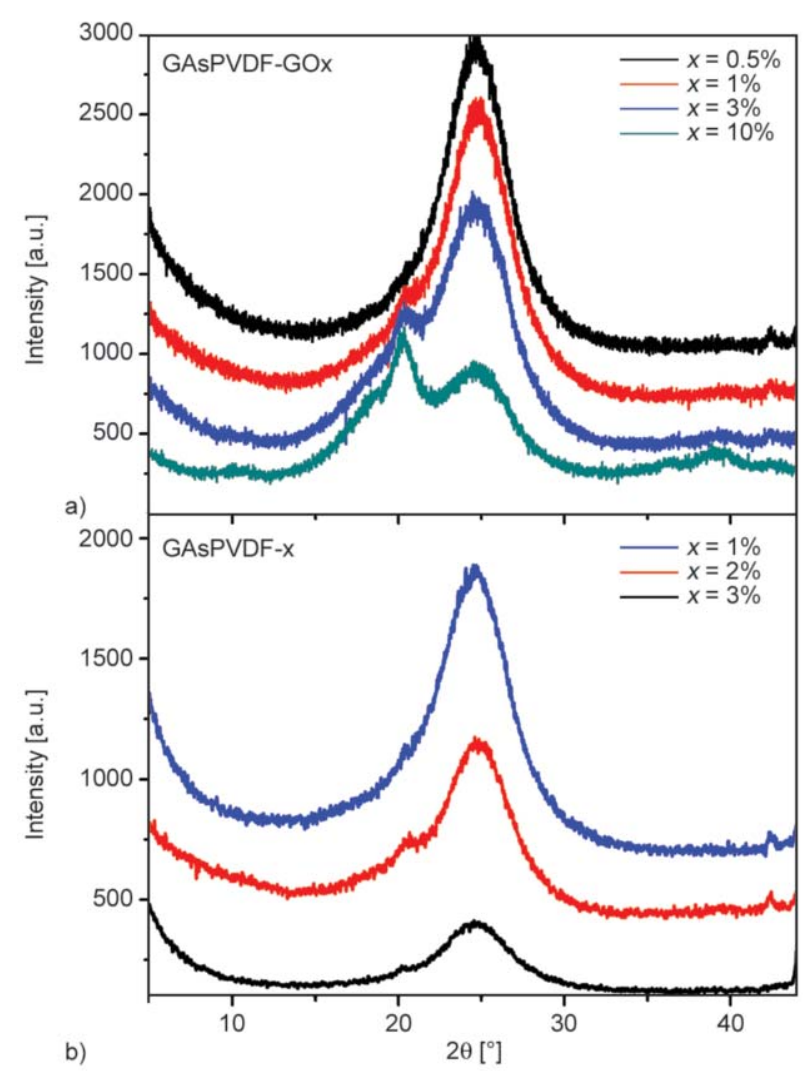

Figure 5. XRD patterns of (a) GAsPVDF-GOx, and (b) GAsPVDF-x samples

due to the attachments of the $-\mathrm{CF}_{2}$ groups of PVDF on the graphene sheets, as illustrated in Figure 1. Therefore the Raman spectra further demonstrate that in the GAsPVDF-GOx samples PVDF is well coated on the RGO or GO sheets inside GAs.

It is well known that the PVDF crystalline $\beta$ phase has excellent piezoelectric and pyroelectric properties [18]. From the characterizations described above, GAsPVDF-GOx prepared from PVDF solution with GO blending obviously have better quality of PVDF

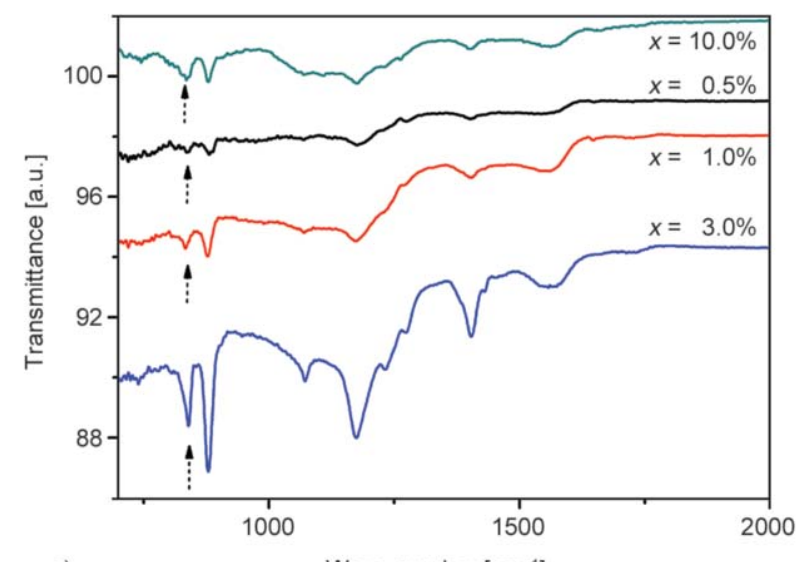

a)

$$
\text { Wave number }\left[\mathrm{cm}^{-1}\right]
$$

coating for piezoelectric and pyroelectric applications. The formation of well coated PVDF films on the graphene sheets in the GAsPVDF-GOx sample can be explained as follows: First, because the GOs are abundant of $-\mathrm{C}=\mathrm{O}$ groups, and GOs and GA exhibit high affinity, therefore the strong interaction between $-\mathrm{C}=\mathrm{O}$ groups in $\mathrm{GOs}$ and $-\mathrm{CF}_{2}$ groups in PVDF drives the PVDF to lay on the graphene sheets inside GA. Second, such interaction promotes the ordering of $-\mathrm{CF}_{2}$ groups in PVDF, resulting in the PVDF films uniformly coated on the graphene sheets inside GAs. Therefore GOs are not only surfactants of GA but also blending elements of PVDF which improve the piezoelectric and ferroelectric properties of PVDF. Interestingly, it has been observed that GOs in de-ionized water actually deteriorate the 3D graphene structures of GA, instead of acting as a surfactant of GA when it is in DMF. Therefore, it is suggested that GO and PVDF first form a nanocomposite which could relieve the oxidation reaction between GO and GA.

\subsection{The mechanical properties of GA supported poly(vinylidene fluoride)}

Figure 7 shows the compressive curves of GAsPVDFGOx and GAsPVDF-x samples, in comparison with that of GA shown in the inset of Figure 7a. The GAsPVDF-GOx and GAsPVDF-x samples exhibit significantly improved mechanical strength as compared with that of GA samples. Remarkably, the mechanical strength of the GAsPVDF-x samples prepared from PVDF solution without GO blending is lower than that of the GAsPVDF-GOx samples prepared from PVDF solution with GO blending, and

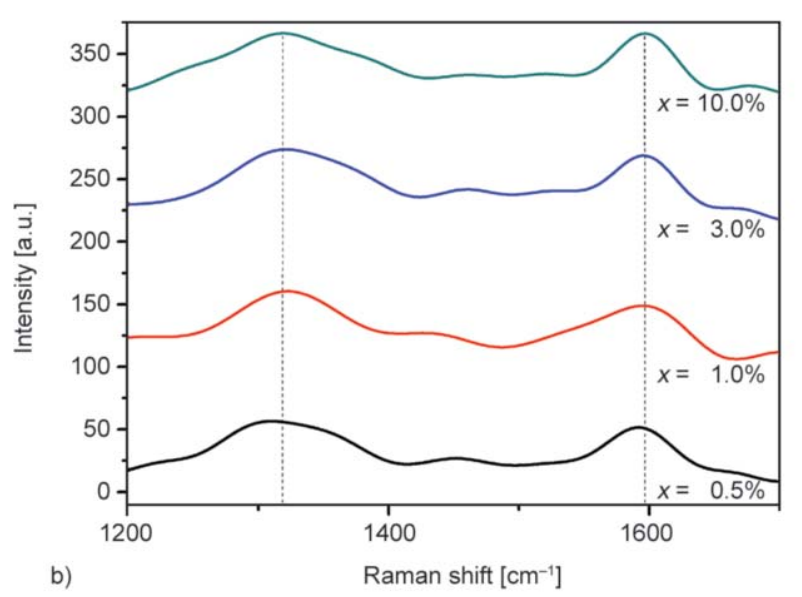

Figure 6. (a) FTIR spectra of GAsPVDF-GOx samples. The arrows indicate the IR transmission peak at $839 \mathrm{~cm}^{-1} \mathrm{which}$ characterizes the PVDF crystalline beta phase. (b) Raman spectra of GAsPVDF-GOx samples. 

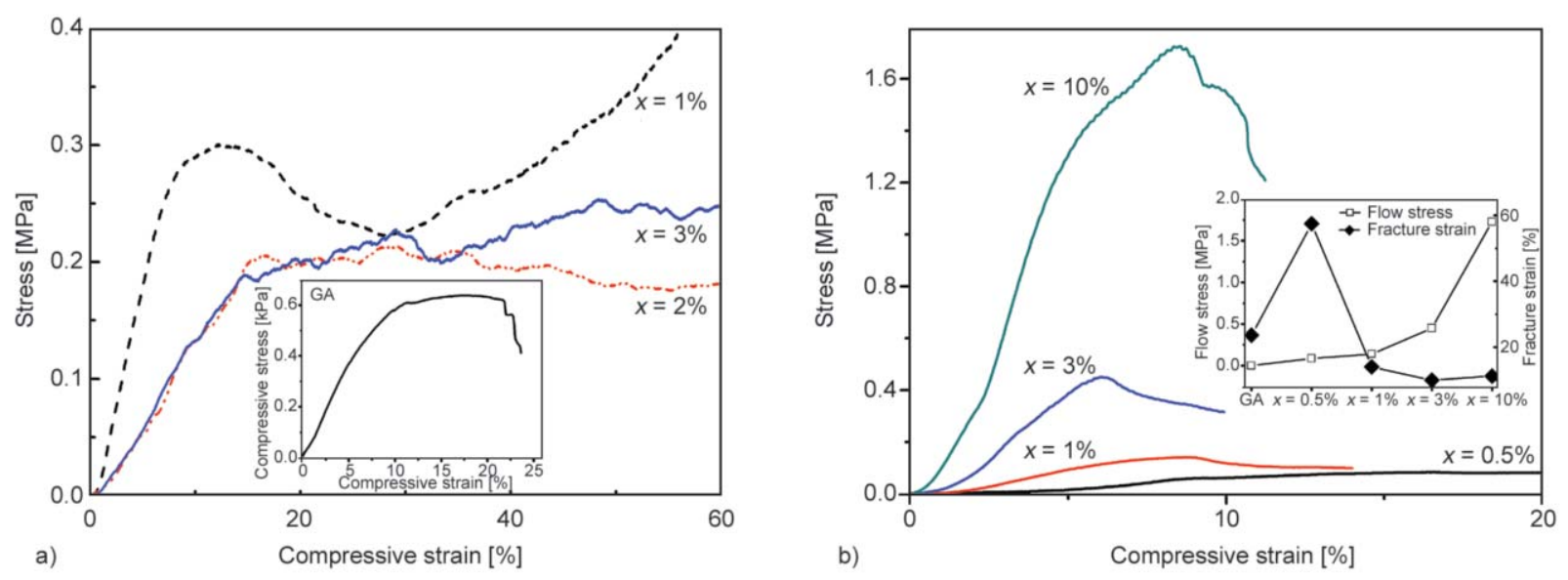

Figure 7. (a) The compressive curves of GAsPVDF-x samples; the inset is that of GA. (b) The compressive curves of GAsPVDF-GOx samples. The inset shows the flow stress and fracture strain of the samples.

is less dependent on the absorbed PVDF content $\mathrm{x}$. As shown in Figure 7b, the mechanical strength of the GAsPVDF-GOx samples prepared from PVDF solution with GO blending increases significantly with increasing PVDF content, suggesting the good and firm coating of PVDF in the 3D graphene structures of GA. On the contrary, the PVDF coating on the graphene sheets in the GAsPVDF-x samples might be loose and not uniform, resulting in the low flow stress which is not much affected by the content of absorbed PVDF.

The effect of PVDF content $x$ on the mechanical strength and ductility of GAsPVDF-GOx is significant, as shown in the inset of Figure $7 \mathrm{~b}$. The flow stress $(0.087 \mathrm{MPa})$ and the fracture strain $(57.5 \%)$ of GAsPVDF-GOx with $x=0.5 \%$ are all much higher than those of pristine GA, suggesting that the PVDF coating on the graphene sheets inside GA is effective in improving the mechanical strength and superplasticity of GA. The enhanced mechanical properties of the GAsPVDF-GOx samples prepared from PVDF solution with GO blending are attributed to the strong interaction between PVDF and GA.

\subsection{The electro-mechanical properties of GA supported poly(vinylidene fluoride)}

The change of electrical resistance $\Delta R$ (absolute value) of the GAsPVDF-GOx samples under applied compressive strain $\varepsilon$ is shown in Figure 8. A linear relation between $\Delta R / R$ and $\varepsilon$ under loading and unloading conditions can be observed. $R$ is the electrical resistance at $\varepsilon=0$ and varies from $1.5 \Omega / \mathrm{cm}^{2}$ for GA to $0.4 \mathrm{M} \Omega / \mathrm{cm}^{2}$ for GAsPVDF-GO10\%. The conductivities of GAsPVDF-GOx are more sensitive to

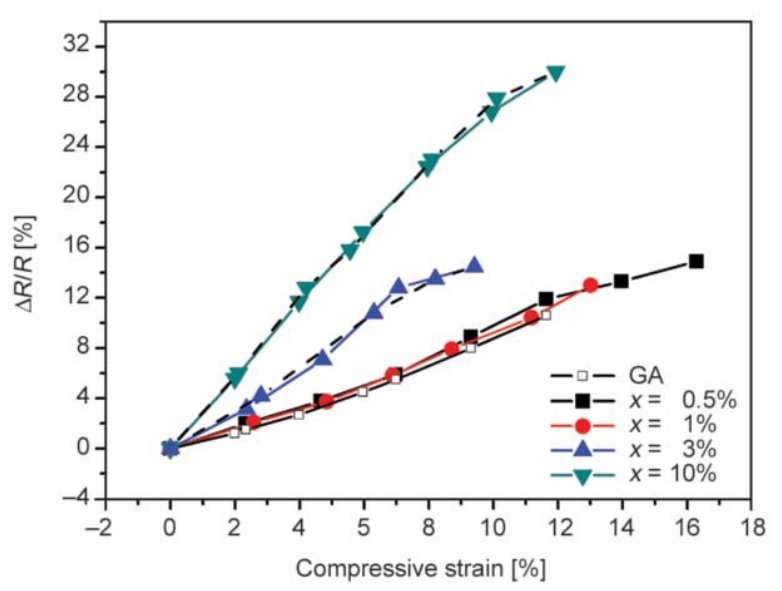

Figure 8. The relative changes of resistances $\Delta R / R$ of GAsPVDF-GOx samples $(x=0.5,1,3$ and $10 \%)$ and GA under compressive strains (in colored solid lines). The $\Delta R / R$ under the release of compression on the samples ( $x=3$ and $10 \%$ ) are plotted in black dash lines

the compressive strain than that of GA. For the GAsPVDF-GO10\% sample prepared from PVDF solution with GO blending, $\Delta R / R$ is about $27 \%$ at $\varepsilon=$ $10 \%$, which is 2.6 times larger than that of GA. As shown in Figure 8, the conductivity of GAsPVDFGOx with more absorbed PVDF is more sensitive to the mechanical compression.

In $\mathrm{GA}$, the dependence of $\Delta R / R$ on $\varepsilon$ is mainly caused by the weakly connected graphene sheets among which the junction areas have low conductivities. Under compression, the volume fraction of the interconnecting graphene sheets increases, resulting in the increase of conductivity with increasing compressive strains. Since the PVDF is an electrical insulator, the effect of PVDF on the relation between $\Delta R / R$ and $\varepsilon$ for the GAsPVDF-GOx samples could be complicat- 
ed. In the GAsPVDF-GOx samples with low PVDF content $(x=1,2 \%)$, PVDF films are not likely to cover most of the surface areas of graphene sheets. Because the junctions among graphene sheets are more active toward PVDF adsorption, PVDF could be coated on those junction areas in GA, leaving the surface areas of the graphene sheets to be conductive. Since graphene sheets are much more flexible than PVDF, the compression on GAsPVDF-GOx leads to the formation of conductive paths among graphene sheets. Therefore the $\Delta R / R$ of GAsPVDFGOx could be more sensitive to $\varepsilon$ than that of GA where the compression leads to the formation of conductive paths at the junction areas.

It is interesting that the change of $\Delta R / R$ with respect to $\varepsilon$ is more sensitive in the GAsPVDF-GOx samples with more PVDF content $x$. When the content of the adsorbed PVDF in GA is high, the PVDF films tend to cover the surfaces of graphene sheets, as demonstrated by the XPS and EDX results listed in Table 1. If the mechanism of compression-induced conductivity change is the same as those described above, the conductivity of the GAsPVDF-GOx samples with $x>1 \%$ must be less sensitive to compression compared with that of the samples with $x<1 \%$. On the contrary, the conductivities are more sensitive to compression for the GAsPVDF-GOx samples with increasing content of adsorbed PVDF $(x>1 \%)$, as shown in Figure 8. Therefore, the strain-dependent conductivity of the GAsPVDF-GOx samples could not be associated with the compression-induced interconnecting graphene sheets. As discussed in Sec. 3.1, PVDF adsorbed in GA should be piezoelectric because it possesses large volume fraction of crystalline polar crystalline phase. The applied compressive strain could induce polarization and net charges on the electrodes of the GAsPVDF-GOx samples. In the measurement of electrical resistance, there are additional dipolar currents generated by the applied strain besides those caused by the applied voltage. Therefore, the $\Delta R / R$ of the GAsPVDF-GOx samples changes more significantly with the applied strain in comparison with that of GA, and the conductivity is more sensitive to compression in the GAsPVDFGOx samples with more PVDF content $x$.

\subsection{Anelastic analysis on the interfaces between GA and PVDF}

Anelastic mechanical relaxation of GAsPVDF-GOx and GAsPVDF-x samples are measured to further elucidate the effect of PVDF on the microstructural features and the mechanical properties of GAs. Under the applied periodic stresses, the anelastic responses of graphene nano-sheets, amorphous and crystalline regions of PVDF films, and those molecular groups at the interfaces between GA and PVDF can be well characterized by the mechanical loss and dynamical modulus. Figure 9 shows the storage modulus and mechanical loss $(\tan \delta)$ of GA monoliths used for the

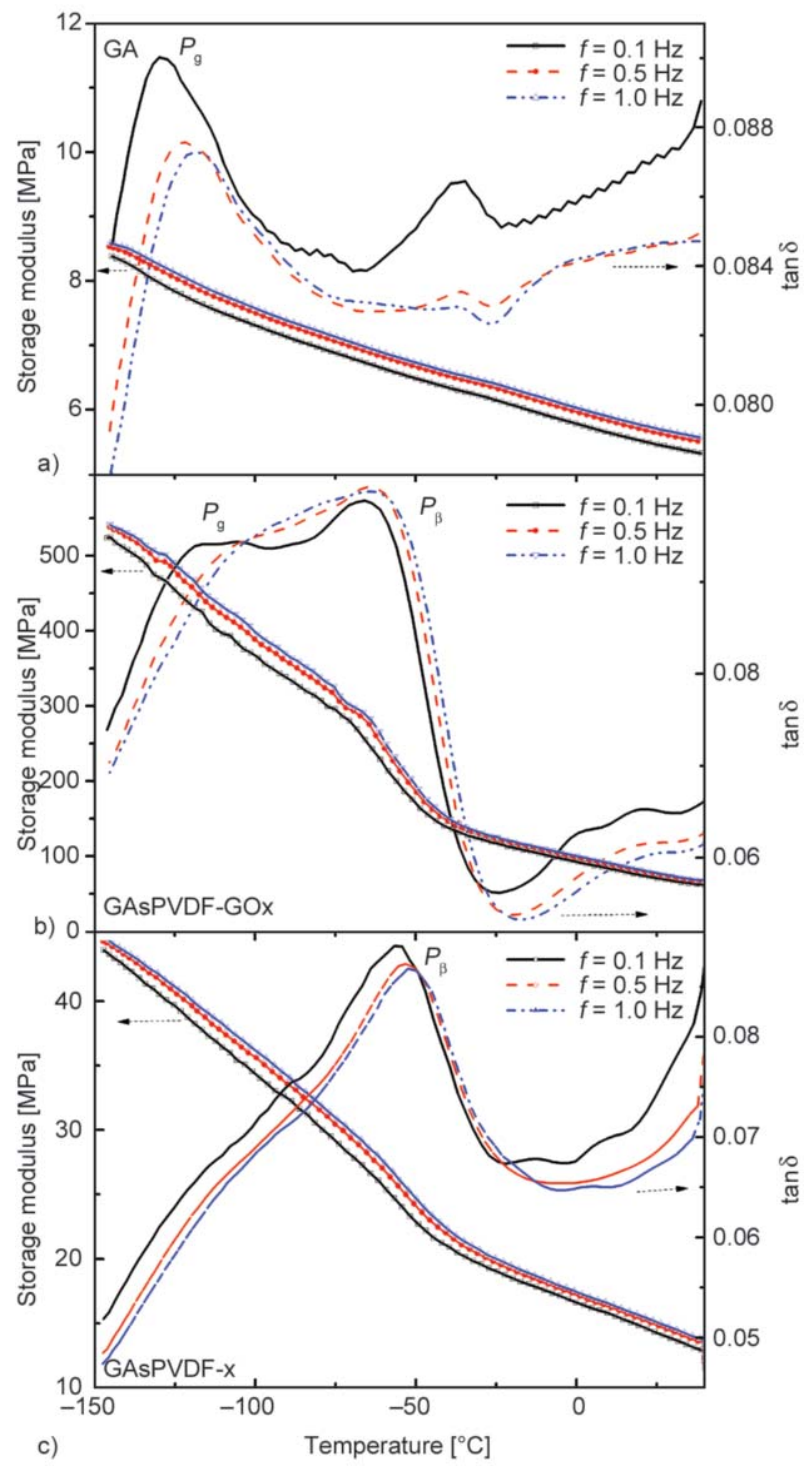

Figure 9. The storage modulus and mechanical losses ( $\tan \delta$ ) of (a) GA, (b) GAsPVDF-GO10\%, and (c) GAsPVDF-10\% samples at various frequencies $f$. 
preparation of GAsPVDF, GAsPVDF-GOx and GAsPVDF-x samples, respectively. The 3D graphene structure in GA is mostly characterized by the $\tan \delta$ peak (labeled as $P_{\mathrm{g}}$ ) at $-130 \sim-120^{\circ} \mathrm{C}$ depending on the testing frequency, which is of relaxation type as shown in Figure 9a. The activation energy of relaxation $E_{\mathrm{a}}$ can be well determined by the Arrhenius relation (Equation (1)):

$f=f_{0} \exp \left(\frac{-E_{\mathrm{a}}}{k_{\mathrm{B}} T}\right)$

where $f$ is the test frequency, $f_{0}$ is the attempt frequency of the diffusion of atoms and $T$ is the peak temperature of $P_{\mathrm{g}}$ peak. $k_{\mathrm{B}}$ is the Boltzmann constant. Figure 10a shows the fits of $\ln f$ and $1 / T$ based on Equation (1). $E_{\mathrm{a}}$ and $f_{0}$ are determined to be $0.38 \mathrm{eV}$ and $2.8 \cdot 10^{11} \mathrm{~s}^{-1}$, respectively. Because the low activation energy $E_{\mathrm{a}}$ is close to that of two-dimensional diffusion of carbon atoms [21], it is suggested that the $P_{\mathrm{g}}$ peak could be related to the relaxation of defective carbon atoms on the graphene sheets in GAs. Considering the nano-porous features of 3D graphene monolith, the relaxation nature of such $\tan \delta$ peak implies that it is caused by the relaxation of the inter-connecting junction areas of graphene sheets in GA where defective carbon atoms exist.

The mechanical losses of GAsPVDF-GO10\% and GAsPVDF-10\% samples are much increased as compared with that of GA. The presence of PVDF films in these samples is much evident from a broad $\beta$ relaxation peak at $-55 \sim-30^{\circ} \mathrm{C}$ (labeled as $P_{\beta}$ ), which is caused by the Brownian motions of amorphous

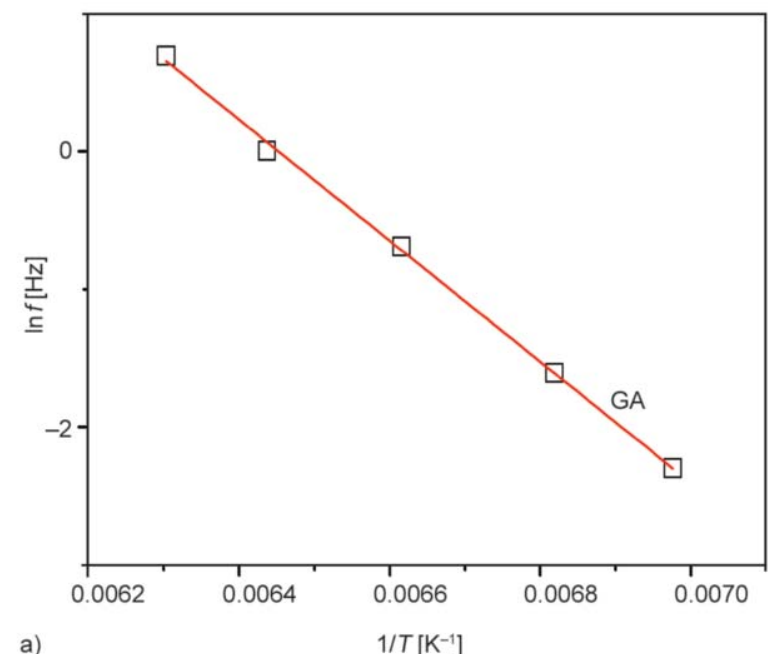

PVDF [22]. In GAsPVDF-10\% samples prepared from PVDF solution without GO blending the $P_{\beta}$ peak seems to overwhelm the characteristic $P_{\mathrm{g}}$ peak of GA. However, in GAsPVDF-GO10\% samples prepared from PVDF solution with GO blending the $P_{\beta}$ peak of PVDF does not affect much the characteristic $P_{\mathrm{g}}$ peak of GA. It is thus suggested that in GAsPVDF-GO10\% samples prepared from PVDF solution with GO blending the $3 \mathrm{D}$ graphene microstructures are mostly preserved since PVDF is coated on the graphene sheets; while in GAsPVDF-10\% samples prepared from PVDF solution without GO blending the 3D graphene microstructures might be damaged by the PVDF deposited at the junction areas of the graphene sheets. In the GAsPVDF-10\% samples amorphous PVDF polymer is dominant in the graphene-PVDF hybrid structures at the inter-connecting regions of GAs.

Unlike those caused by the diffusions of defective carbon atoms, the mechanical relaxation of amorphous structures ( $\beta$ relaxation) in PVDF cannot be characterized by the Arrhenius relation. Instead the $\beta$ relaxation is typically characterized by the Vogel-FulcherTammann (VFT) relation as shown by Equation (2):

$f=f_{0} \exp \left[\frac{-E_{\mathrm{a}}}{k_{\mathrm{B}}\left(T-T_{0}\right)}\right]$

where $T$ is the peak temperature of $P_{\beta}$ peak. $T_{0}$ represents the Kauzmann temperature or static freezing temperature which is far below the glass transition temperature. Figure 10b shows the fits of $\ln f$ and $1 /\left(T-T_{0}\right)$ based on Equation (2). $T_{0}=-140^{\circ} \mathrm{C}$ is chosen for the fits. The apparent activation energy $E_{\mathrm{a}}$ of

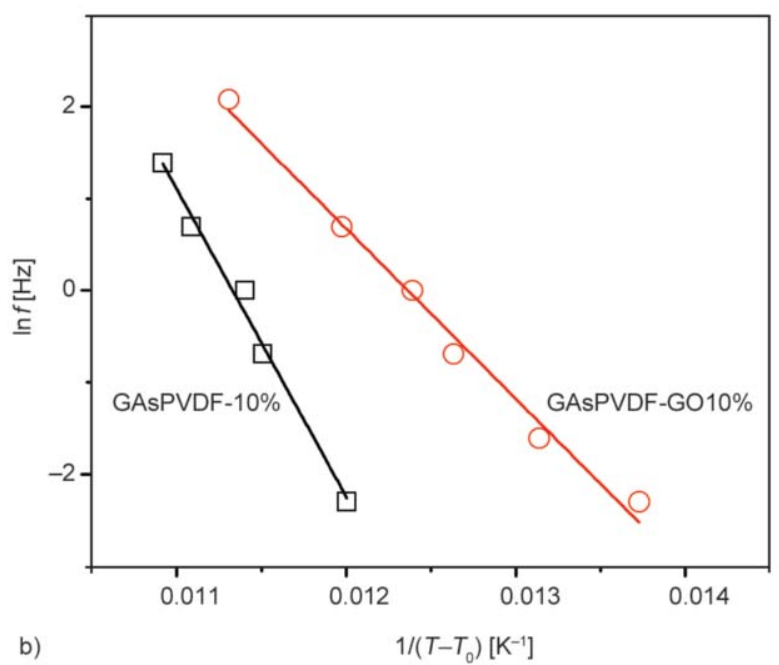

Figure 10. (a) The Arrhenius plots for the $P_{\mathrm{g}}$ peak in GAs. (b) Determination of the apparent activation energy of $\beta$ relaxation using the VFT relation. 
$\beta$ relaxation for the GAsPVDF-GO10\% and GAsPVDF- $10 \%$ samples is $0.16 \mathrm{eV}$ and $0.29 \mathrm{eV}$, respectively. Because $E_{\mathrm{a}}$ of $\beta$ relaxation for the GAsPVDF-GO10\% sample is consistent with that $(\sim 0.15 \mathrm{eV})$ for PVDF-GO composite films [23] and is different with that $(\sim 0.09 \mathrm{eV})$ for pure PVDF films [18], it is envisaged that GOs are incorporated into the PVDF films in GAsPVDF-GO10\% samples. Since the additions of GOs in PVDF enhance the polar crystalline phase of PVDF [17, 18, 23], the films in GAsPVDF-GOx samples have less amorphous structures than those in GAsPVDF-x samples. Comparison between the $P_{\beta}$ peak temperatures for the GAsPVDF-GO10\% samples and the GAsPVDF-10\% samples could provide further evidence, as shown in Figure $9 \mathrm{~b}$ and $9 \mathrm{c}$, respectively. Nevertheless, $E_{\mathrm{a}}$ of $\beta$ relaxation for the GAsPVDF-10\% samples is much larger than that for the GAsPVDF-GO10\% samples, demonstrating the high content of amorphous structures in PVDF which prevents the formation of well coated PVDF films on the graphene surfaces in GAs, as shown in Figure 10b.

\section{Conclusions}

Graphene aerogel supported poly(vinylidene fluoride) samples were synthesized. The blending of GOs with PVDF solutions facilitates the formation of thin and uniform PVDF films whose typical thickness is below $100 \mathrm{~nm}$ on the graphene sheets inside GAs. The graphene aerogel supported poly(vinylidene fluoride) has high content of crystalline $\beta$ phase which possesses pyroelectricity and piezoelectricity. With increasing contents of PVDF and crystalline PVDF $\beta$ phase, the graphene aerogel supported poly(vinylidene fluoride) samples exhibit significantly improved mechanical and electro-mechanical properties as compared to those of GAs, suggesting that they could be used as multi-functional materials such as highperformance sensors, actuators and kinetic energy harvesters. The superior combined mechanical and electro-mechanical properties of graphene aerogel supported poly(vinylidene fluoride) are attributed to the strong interaction between the $-\mathrm{C}=\mathrm{O}$ groups in GAs or GOs and the $-\mathrm{CF}_{2}$ groups in PVDF.

\section{Acknowledgements}

The authors are grateful for the supports provided by the Science and Technology Innovation Commission of Shenzhen (No. JCYJ2013041152508657), and the Program for Eastern Scholar at Shanghai Institutions of Higher Learning.

\section{References}

[1] Hu H., Zhao Z., Wan W., Gogotsi Y., Qiu J.: Ultralight and highly compressible graphene aerogels. Advanced Materials, 25, 2219-2223 (2013).

DOI: $10.1002 / \mathrm{adma} .201204530$

[2] Sun H., Xu Z., Gao C.: Multifunctional, ultra-flyweight, synergistically assembled carbon aerogels. Advanced Materials, 25, 2554-2560 (2013).

DOI: $10.1002 / \mathrm{adma} .201204576$

[3] Ito Y., Tanabe Y., Qiu H. J., Sugawara K., Heguri S., Tu N. H., Huynh K. K., Fujita T., Takahashi T., Tanigaki K., Chen M. W.: High-quality three-dimensional nanoporous graphene. Angewandte Chemie, 126, 4922-4926 (2014). DOI: 10.1002 /ange.201402662

[4] Biener J., Dasgupta S., Shao L., Wang D., Worsley M. A., Wittstock A., Lee J. R., Biener M. M., Orme C. A., Kucheyev S. O., Wood B. C., Willey T. M., Hamza A. V., Weissmüller J., Hahn H., Baumann T. F.: Macroscopic 3D nanographene with dynamically tunable bulk properties. Advanced Materials, 24, 5083-5087 (2012). DOI: $10.1002 / \mathrm{adma} .201202289$

[5] Gui X., Cao W., Wei J., Li H., Jia Y., Li Z., Fan L., Wang K., Zhu H., Wu D.: Soft, highly conductive nanotube sponges and composites with controlled compressibility. ACS Nano, 4, 2320-2326 (2010).

DOI: $10.1021 / \mathrm{nn} 100114 \mathrm{~d}$

[6] Zhang X., Sui Z., Xu B., Yue S., Luo Y., Zhan W., Liu B.: Mechanically strong and highly conductive graphene aerogel and its use as electrodes for electrochemical power source. Journal of Materials Chemistry, 21, 6494 6497 (2011).

DOI: $10.1039 / \mathrm{C} 1 \mathrm{JM} 10239 \mathrm{G}$

[7] Feng W., Yan L.: In situ self-assembly of mild chemical reduction graphene for three-dimensional architectures. Nanoscale, 3, 3132-3137 (2011). DOI: $10.1039 / \mathrm{C} 1 \mathrm{NR} 10355 \mathrm{E}$

[8] Qiu L., Liu J. Z., Chang S. L. Y., Wu Y., Li D.: Biomimetic superelastic graphene-based cellular monoliths. Nature Communications, 3, 1241/1-1241/7 (2012). DOI: $10.1038 /$ ncomms 2251

[9] Hodlur R. M., Rabinal M. K.: Self assembled graphene layers on polyurethane foam as a highly pressure sensitive conducting composite. Composites Science and Technology, 90, 160-165 (2014). DOI: $10.1016 /$ j.compscitech.2013.11.005 
[10] Kuang J., Liu L., Gao Y., Zhou D., Chen Z., Han B., Zhang Z.: A hierarchically structured graphene foam and its potential as a large-scale strain-gauge sensor. Nanoscale, 5, 12171-12177 (2013).

DOI: $10.1039 / \mathrm{C} 3 \mathrm{NR} 03379 \mathrm{~A}$

[11] Rahman M. A., Lee B. C., Phan D. T., Chung G. S.: Fabrication and characterization of highly efficient flexible energy harvesters using PVDF-graphene nanocomposites. Smart Materials and Structures, 22, 085017/1085017/5 (2013).

DOI: $10.1088 / 0964-1726 / 22 / 8 / 085017$

[12] Cong H-P., Ren X-C., Wang P., Yu S-H.: Macroscopic multifunctional graphene-based hydrogels and aerogels by a metal ion induced self-assembly process. ACS Nano, 6, 2693-2703 (2012). DOI: $10.1021 / \mathrm{nn} 300082 \mathrm{k}$

[13] Hu H., Zhao Z., Zhang R., Bin Y., Qiu J.: Polymer casting of ultralight graphene aerogels for the production of conductive nanocomposites with low filling content. Journal of Materials Chemistry A, 2, 3456-3760 (2014). DOI: $\underline{10.1039 / \mathrm{C} 3 \mathrm{TA} 14840 \mathrm{H}}$

[14] Hu H., Zhao Z., Wan W., Gogotsi Y., Qiu J.: Polymer/ graphene hybrid aerogel with high compressibility, conductivity, and 'sticky' superhydrophobicity. ACS Applied Materials and Interfaces, 6, 3242-3249 (2014). DOI: 10.1021/am4050647

[15] Gong Y., Yang S., Liu Z., Ma L., Vajtai R., Ajayan P. M.: Graphene-network-backboned architectures for high-performance lithium storage. Advanced Materials, 25, 3979-3984 (2013). DOI: $10.1002 / \mathrm{adma} .201301051$

[16] Xiao L., Wu D., Han S., Huang Y., Li S., He M., Zhang F., Feng X.: Self-assembled $\mathrm{Fe}_{2} \mathrm{O}_{3}$ /graphene aerogel with high lithium storage performance. ACS Applied Materials and Interfaces, 5, 3764-3769 (2013).

DOI: $10.1021 / \mathrm{am} 400387 \mathrm{t}$
[17] El Achaby M., Arrakhiz F. Z., Vaudreuil S., Essassi E. M., Qaiss A.: Piezoelectric $\beta$-polymorph formation and properties enhancement in graphene oxide - PVDF nanocomposite films. Applied Surface Science, 258, 7668-7677 (2012).

DOI: $10.1016 /$ j.apsusc.2012.04.118

[18] Jiang Z. Y., Zheng G. P., Han Z., Liu Y. Z., Yang J. H.: Enhanced ferroelectric and pyroelectric properties of poly(vinylidene fluoride) with addition of graphene oxides. Journal of Applied Physics, 115, 204101/1204101/6 (2014).

DOI: $10.1063 / 1.4878935$

[19] Ilčíková M., Mrlík M., Sedláček T., Doroshenko M., Koynov K., Danko M., Mosnáček J.: Tailoring of viscoelastic properties and light-induced actuation performance of triblock copolymer composites through surface modification of carbon nanotubes. Polymer, 72, 368-377 (2015).

DOI: $10.1016 /$ j.polymer.2015.03.060

[20] Han Z., Tang Z., Shen S., Zhao B., Zheng G. P., Yang J. H.: Strengthening of graphene aerogels with tunable density and high adsorption capacity towards $\mathrm{Pb}^{2+}$. Scientific Reports, 4, 5025/1-5025/6 (2014). DOI: $10.1038 /$ srep05025

[21] Hofmann S., Csányi G., Ferrari A. C., Payne M. C., Robertson J.: Surface diffusion: The low activation energy path for nanotube growth. Physical Review Letters, 95, 036101/1-036101/4 (2005).

DOI: $10.1103 /$ PhysRevLett.95.036101

[22] Callens A., Eersels L., De Batist R.: Low temperature internal friction on $\gamma$-irradiated polyvinylidene fluoride (PVDF). Journal of Materials Science, 13, 1887-1990 (1978).

DOI: $10.1007 / \mathrm{BF} 00552895$

[23] Jiang Z. Y., Zheng G. P., Zhan K., Han Z., Yang J. H.: Formation of piezoelectric $\beta$-phase crystallites in poly (vinylidene fluoride)-graphene oxide nanocomposites under uniaxial tensions. Journal of Physics D: Applied Physics, 48, 245303/1-245303/7 (2015). DOI: $\underline{10.1088 / 0022-3727 / 48 / 24 / 245303}$ 